Research paper

\title{
Polarity inversion of bioanode for biocathodic reduction of aromatic pollutants
}

\author{
Hui Yun ${ }^{\mathrm{a}, \mathrm{b}}$, Bin Liang ${ }^{\mathrm{a}, *}$, De-Yong Kong ${ }^{\mathrm{c}, \mathrm{d}}$, Hao-Yi Cheng ${ }^{\mathrm{a}}$, Zhi-Ling Li $^{\mathrm{d}}$, Ya-Bing Gu ${ }^{\mathrm{e}}$, \\ Hua-Qun Yin ${ }^{\mathrm{e}}$, Ai-Jie Wang ${ }^{\mathrm{a}, \mathrm{d}, * *}$ \\ a Key Laboratory of Environmental Biotechnology, Research Center for Eco-Environmental Sciences, Chinese Academy of Sciences, Beijing 100085, China \\ ${ }^{\mathrm{b}}$ University of Chinese Academy of Sciences, Beijing, China \\ ' Shenyang Academy of Environmental Sciences, Shenyang 110167, China \\ d State Key Laboratory of Urban Water Resource and Environment, Harbin Institute of Technology, Harbin 150090, China \\ ${ }^{\text {e }}$ Key Laboratory of Biometallurgy of Ministry of Education, School of Minerals Processing and Bioengineering, Central South University, Changsha 410083, \\ China
}

\section{H I G H L I G H T S}

- Biocathode was rapidly developed by polar inversion of bioanode.

- Biocathode could accelerate reduction of nitroaromatic and azo dye.

- The dominant bacteria possess bidirectional electron transfer capability.

- Biocathode could be modularly established for wastewater treatment.

\section{A R T I C L E I N F O}

\section{Article history:}

Received 18 December 2016

Received in revised form 7 February 2017

Accepted 26 February 2017

Available online 27 February 2017

\section{Keywords:}

Polarity inversion

Bidirectional electron transfer

Biocathode

Nitroaromatics and azo dye reduction

\begin{abstract}
A B S T R A C T
The enrichment of specific pollutant-reducing consortium is usually required prior to the startup of biocathode bioelectrochemical system (BES) and the whole process is time consuming. To rapidly establish a non-specific functional biocathode, direct polar inversion from bioanode to biocathode is proposed in this study. Based on the diverse reductases and electron transfer related proteins of anode-respiring bacteria (ARB), the acclimated electrochemically active biofilm (EAB) may catalyze reduction of different aromatic pollutants. Within approximately $12 \mathrm{~d}$, the acclimated bioanodes were directly employed as biocathodes for nitroaromatic nitrobenzene (NB) and azo dye acid orange 7 (AO7) reduction. Our results indicated that the established biocathode significantly accelerated the reduction of NB to aniline (AN) and $\mathrm{AO} 7$ to discolored products compared with the abiotic cathode and open circuit controls. Several microbes possessing capabilities of nitroaromatic/azo dye reduction and bidirectional electron transfer were maintained or enriched in the biocathode communities. Cyclic voltammetry highlighted the decreased over-potentials and enhanced electron transfer of biocathode as well as demonstrated the ARB Geobacter containing cytochrome $c$ involved in the backward electron transfer from electrode to NB. This study offers new insights into the rapid establishment and modularization of functional biocathodes for the potential treatment of complicated electron acceptors-coexisting wastewaters.
\end{abstract}

(c) 2017 Elsevier B.V. All rights reserved.

\footnotetext{
* Corresponding author at: Key Laboratory of Environmental Biotechnology, Research Center for Eco-Environmental Sciences, Chinese Academy of Sciences, Beijing 100085, China.

** Corresponding author.

E-mail addresses: binliang@rcees.ac.cn (B. Liang), ajwang@rcees.ac.cn (A.-J. Wang).
}

\section{Introduction}

Bioelectrochemical systems (BES), using electrochemically active microorganisms to catalyze oxidative or reductive reactions in the anode or cathode respectively, have attracted growing attention in recent decades [1-3], especially the reductive metabolism with biocathode [4,5]. Cathodes can provide non-exhaustible electrons for the reduction of diverse organic pollutants, including nitroaromatics, azo dyes, haloaromatics and reducible antibiotics [6-15]. Moreover, the biocathode had significantly higher efficiency and selectivity to pollutants reduction than that of the 
abiotic cathode $[8,16,17]$. Though it is self-renewable and potentially cost-effective, cathode biofilms, to some extent, are hard to establish, particularly under anaerobic conditions. Based on our previous studies, the enrichment of targeted nitroaromaticsreducing consortium needs 3-4 weeks, and then the establishment of biocathode with the functional consortium costs another 2-3 weeks. The whole process to achieve the specific pollutantsreducing biocathode in this way totals at least $5-7$ weeks $[8,16,17]$. It is worth mentioning that some electrochemically active bacteria (EAB) are probably eliminated during such non-electrode selection and acclimation stage, for which inevitable co-substrate would potentially enrich more fermentative bacteria.

Some researchers had assumed to improve the biocathode establishment by the bioanode formed firstly, and then inverted it to the cathode for the denitrification, energy generation and oxygen-reducing over-potential decrease [18-22]. Very recently, the EAB of antibiotic chloramphenicol-acclimated bioanode has been proved to carry out the bidirectional electron transfer for chloramphenicol reduction [23]. However, whether the biocathodic reduction of toxic nitro- and azo-aromatics is feasible with directly inverted bioanode is remain poorly understood.

Based on the anodic biofilm acclimation and polar inversion, some EAB can be effectively screened and maintained. These electrode-respiring bacteria, such as Geobacter, could use the electrode as electron donor for the reduction of different pollutants [24]. Under such protocol, the biocathode establishment time (approximately $12 \mathrm{~d}$ ) was obviously decreased, and the developed biocathode communities likely worked on various reducible contaminants by diverse reductases and electron transfer related proteins. The hypothesis of this study is illustrated in Fig. 1. Generally, many kinds of organic or inorganic environmental pollutants coexist in practical wastewater, such as nitro-, halo-aromatics, or dyes in the oxidation state accompanied with nitrate or sulfate. They can work as electron acceptors which accept electrons from the electrode. Such operation has advantages of simplified operation, compressed acclimation period, bidirectional electron transfer ability, efficient functional performance and self-regeneration, which has great potential to integrate with existing BES for the treatment of electron acceptors coexisting wastewaters. Consequently, two hazardous aromatic pollutants were selected to be reduced with this polar inversion strategy. One of the targeted pollutants is nitroaromatic nitrobenzene (NB), which is widely used in industrial and agricultural activities [17]. Acid orange 7 (AO7) is a basic azo dye compound, which has been extensively applied in textile printing and pharmaceutical industries [9]. Large amount of discharges have posed great threaten to public health and diverse ecosystems. The complete removal of these two targeted pollutants could be realized by bioelectrochemical reduction, which the traditional enriched biocathode had been reported as one of valid routes $[16,17,25]$, and followed with aerobic biodegradation [26].

The objectives of this study were to test (i) whether the simple polarity inversion strategy could accelerate the establishment of a non-specific functional biocathode; (ii) whether the established biocathode could possess powerful electron transfer ability and obvious reducing power for NB and AO7 and (iii) whether the polarity inversion could significantly alter the biofilm community structure and composition. The current study offers new insights into the applicative outlook for functional biocathodes modularization in complicated electron acceptors coexisting wastewaters treatment.

\section{Materials and methods}

\subsection{Bioanode acclimation}

The electrochemical reactors were constructed as described previously [6]. The net volume of each chamber was ca. $28 \mathrm{~mL}$. Carbon brushes were used as both anodic and cathodic electrodes ( $2 \mathrm{~cm}$ in diameter by $3 \mathrm{~cm}$ in length, TOHO TENAX Co., Ltd., Tokyo, Japan). They were pretreated as described elsewhere [6]. An external resistor $(10 \Omega)$ was employed within the circuit. A saturated calomel electrode (SCE, 0.247 V vs. standard hydrogen electrode (SHE), model-217, Shanghai Precise. Sci. Instru. Co., Ltd., China) was used for potential acquisition with a data acquisition system (Model 2700, Keithley Instruments Inc., U.S.). All reported potentials herein were expressed against the SHE. Five mililiter anaerobic sludge from Taiping wastewater treatment plant (Harbin, China) worked as the anodic inoculum. Medium composition and preparation of the catholyte and anolyte containing $10 \mathrm{mM}$ phosphate buffer solution (PBS) were described previously [27]. The solution in the two chambers were refreshed every 3 days. After 3-4 times replacing, the mature bioanodes would be established with the stable voltage output as an indicator.

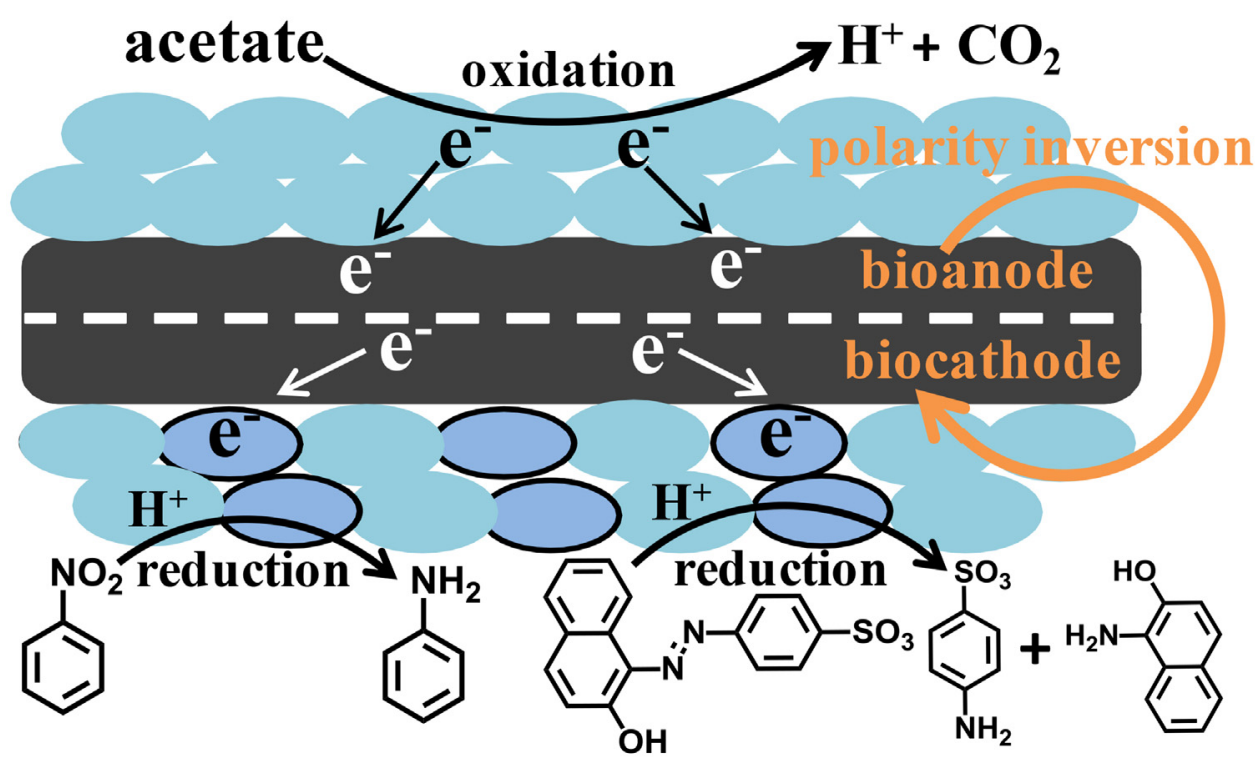

Fig. 1. Hypothesis of direct polarity inversion of bioanode for biocathodic reduction of aromatic pollutants. 


\subsection{Inversion of conventional bioanode to biocathode}

After the anode biofilms were well developed, half of anode brushes with similar polarization characteristics were inverted to cathodes, while another half were still worked as bioanodes. The oxygen-free electrolyte amended with $20 \mathrm{mM}$ sodium acetate was filled in both anode and cathode chambers, and the catholyte synchronously supplied with NB or AO7, unless otherwise stated. The established biocathode and abiotic cathode (without inoculum) were poised at the same potential of -0.45 or $-0.40 \mathrm{~V}$ by a potentiostat (model-CHI 1000C, CHI Instruments Inc. U.S.). For the NB reduction, the initial NB concentration was $0.25 \mathrm{mM}$ for 3 cycles. And then the NB concentration was increased to $0.50 \mathrm{mM}$. After NB experiments, $0.143 \mathrm{mM}$ AO7 took place of NB and worked as electron acceptor. Conditions of catholyte with (ace-biocathode) and without sodium acetate (with $10 \mathrm{mM} \mathrm{NaHCO}_{3}$ as carbon source and electrode as electron donor, namely $\mathrm{CO}_{2}$-biocathode), abiotic cathode and open circuit biocathode (sodium acetate as carbon source and electron donor) were all tested for NB and AO7 reduction. For the open circuit biocathode, the circuit was unlinked to show the pure anaerobic bioreduction activity. For each condition, at least 3 cycles were carried out in triplicate. All reactors were operated at ambient temperature $\left(23 \pm 3^{\circ} \mathrm{C}\right)$.

\subsection{Chemicals and analytical methods}

NB ( $\geq 99.8 \%$ purity), aniline (AN, $\geq 99.9 \%$ purity), AO7 ( $\geq 98 \%$ purity), and sulfanilic acid (SA, $\geq 99.5 \%$ purity) were purchased from Aladdin (Shanghai, China). Methanol (HPLC grade) and nitrosobenzene (NOB, $\geq 97 \%$ purity) were purchased from Sigma-Aldrich (St. Louis, MO, U.S.). Other chemicals used were of analytical reagent grade.

Samples taken from each reactor were filtered through $0.22 \mu \mathrm{m}$ filters (Shanghai XingYa Purification Material Co., China) and stored at $4{ }^{\circ} \mathrm{C}$ less than $24 \mathrm{~h}$. NB, AN, NOB, AO7, and SA were determined as described previously $[9,17]$. The current calculation, the targeted pollutants reduction $\left(\mathrm{E}_{\mathrm{rNB}}\right.$ and $\left.\mathrm{E}_{\mathrm{rAO}}\right)$ and corresponding products formation efficiencies $\left(E_{A N}\right.$ and $\left.E_{S A}\right)$ as well as the coulombic efficiency $(\mathrm{CE})$ for the cathodic reduction reactions were calculated as reported previously $[9,12]$. The rates of substrates reduction and products formation were fitted using an apparent first-order reaction model as described elsewhere [16].

\subsection{Cyclic voltammetry (CV) analysis}

To improve the CV scan sensitivity, a same reactor was prepared and operated except that carbon cloth $3 \mathrm{~cm}$ in diameter,
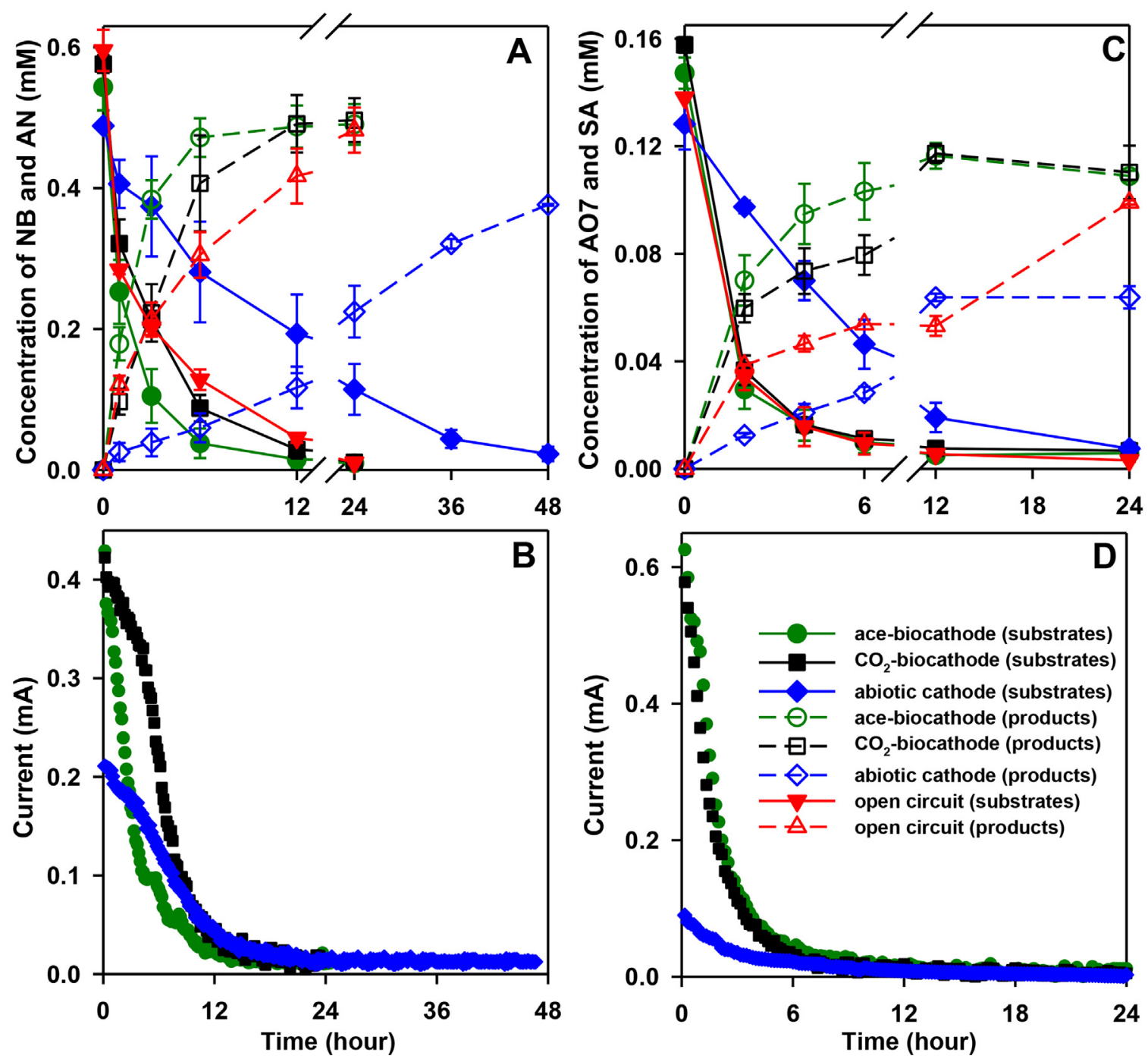

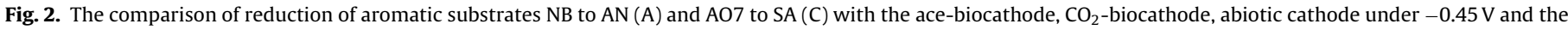
open circuit biocathode. The reaction currents of reduction of NB (B) and AO7 (D) were also shown. 
Table 1

NB and AO7 reduction efficiencies (\%) under different operational conditions.

\begin{tabular}{|c|c|c|c|c|c|c|c|c|}
\hline & $\mathrm{E}_{\mathrm{rNB}-6 \mathrm{~h}}$ & $\mathrm{E}_{\mathrm{AN}-6 \mathrm{~h}}$ & $\mathrm{E}_{\mathrm{rNB}-24 \mathrm{~h}}$ & $\mathrm{E}_{\mathrm{AN}-24 \mathrm{~h}}$ & $\mathrm{E}_{\mathrm{rAO} 7-6 \mathrm{~h}}$ & $\mathrm{E}_{\mathrm{SA}-6 \mathrm{~h}}$ & $\mathrm{E}_{\mathrm{rAO} 7-24 \mathrm{~h}}$ & $\mathrm{E}_{\mathrm{SA}-24 \mathrm{~h}}$ \\
\hline ace-biocathode & $92.4 \pm 4.5^{\mathrm{a}}$ & $84.5 \pm 5.3^{a}$ & $97.5 \pm 2.6^{a}$ & $92.5 \pm 5.0^{\mathrm{a}}$ & $94.3 \pm 2.1^{\mathrm{a}}$ & $74.2 \pm 6.0^{\mathrm{a}}$ & $96.5 \pm 0.9^{a}$ & $80.2 \pm 0.4^{\mathrm{a}}$ \\
\hline $\mathrm{CO}_{2}$-biocathode & $84.7 \pm 4.7^{\text {be }}$ & $77.2 \pm 8.0^{\mathrm{ab}}$ & $98.0 \pm 1.5^{\mathrm{a}}$ & $90.3 \pm 2.4^{\mathrm{a}}$ & $92.9 \pm 0.8^{\mathrm{ab}}$ & $51.8 \pm 5.5^{\mathrm{b}}$ & $95.9 \pm 0.8^{\mathrm{ab}}$ & $74.8 \pm 5.4^{\mathrm{ab}}$ \\
\hline abiotic cathode & $42.5 \pm 9.8^{c}$ & $28.7 \pm 2.2^{c}$ & $76.6 \pm 7.4^{\mathrm{b}}$ & $59.9 \pm 6.1^{\mathrm{b}}$ & $68.9 \pm 6.1^{c}$ & $37.6 \pm 2.4^{c}$ & $95.4 \pm 1.7^{\mathrm{ac}}$ & $72.1 \pm 4.1^{\mathrm{ac}}$ \\
\hline open circuit & $77.9 \pm 2.6^{\text {de }}$ & $65.7 \pm 3.1^{\text {bd }}$ & $98.1 \pm 1.6^{\mathrm{a}}$ & $84.9 \pm 4.7^{\mathrm{a}}$ & $91.8 \pm 2.4^{\mathrm{ad}}$ & $42.7 \pm 0.9^{\mathrm{cd}}$ & $97.7 \pm 0.4^{c}$ & $73.5 \pm 4.1^{\mathrm{ac}}$ \\
\hline
\end{tabular}

Group with the same letters are not significantly different from each other at the 0.05 level.

nonwetproof, YB-20, YiBang Technology Co., Ltd., China) replaced the graphite carbon brush. Before scanning, the NB reduction efficiency was measured to ensure the biofilm catalytic function. An electrochemical workstation (model-660D, CHI Instruments Inc., U.S.) equipped with three-electrode system was used. The carbon cloth with/without biofilm and inactivated biofilm (sterilized at $121^{\circ} \mathrm{C}$ for $1 \mathrm{~h}$ for twice) worked as working electrodes, while a sterilized carbon brush and an SCE as counter electrode and reference electrode, respectively. Cyclic voltammograms were recorded at ambient temperature with a scan rate of 2 or $10 \mathrm{mV} / \mathrm{s}$.

\subsection{Microbial community analysis}

Three anode and five cathode carbon brushes were randomly sampled with a pair of aseptic scissors and transferred into sterile tubes filled with appropriate sterilized $10 \mathrm{mM}$ PBS. The microbes were shaken down with a vortex and collected by centrifugation (13000 $\mathrm{g}$ for $20 \mathrm{~min}$ ). The details for the genomic DNA extraction, 16S rRNA gene PCR amplification and Illumina MiSeq sequencing as well as data analysis according to a previous study [39].

\section{Results and discussion}

\subsection{NB reduction with the established biocathode}

After the successful establishment of the bioanode, the anodic potential could reach $-0.25 \mathrm{~V}$, and therefore, the established bioanodes were directly employed as biocathodes and poised at $-0.45 \mathrm{~V}$. In order to make a short acclimation to $\mathrm{NB}$, the initial NB concentration was $0.25 \pm 0.01 \mathrm{mM}$. The established biocathode could transform $94.1 \pm 5.4 \% \mathrm{NB}$ with AN formation efficiency of $90.6 \pm 0.5 \%$ within $6 \mathrm{~h}$. The intermediate NOB was detected only in the initial $1 \mathrm{~h}$ and phenylhydroxylamine was undetected throughout the process, which suggested the well-developed cathode biofilms had stable NB reduction performance. After 3 cycles $(24 \mathrm{~h}$ for one cycle), the NB concentration was raised to $0.54 \pm 0.03 \mathrm{mM}$ for data collection.

With sodium acetate as carbon and energy sources, NB $(0.54 \pm 0.03 \mathrm{mM})$ was depleted and formed $0.48 \pm 0.04 \mathrm{mM}$ AN at $-0.45 \mathrm{~V}$ within $24 \mathrm{~h}$. The NB to AN transformation efficiency was $92.5 \pm 5.0 \%$, which was not obviously different $(P=0.51)$ from the $\mathrm{CO}_{2}$-biocathode $(90.3 \pm 2.4 \%)$ (Fig. $2 \mathrm{~A}$ and Table 1$)$. This could be explained as some exoelectrogens tend to be electrotrophs, which likely accepted electrons from the electrode to reduce NB. The tiny difference was also in accordance with the current trends, the initial currents of both biocathode modes quickly soared to nearly $0.4 \mathrm{~mA}$, and declined gradually with the removal of NB (Fig. 2B). The trend had recurred once refreshing the solution of the two chambers. Thus, the maximum current-falling was corresponding to the major part of NB loss, in which stage sufficient electron could be continuously supplied. This could show the strong ability of bidirectional electron transfer of the biofilm. The ace-biocathode had significantly higher $\mathrm{E}_{\mathrm{rNB}}(P<0.03$ for $1-6 \mathrm{~h})$ and $\mathrm{E}_{\mathrm{AN}}(P<0.0005$ for $1-3 \mathrm{~h}$ ) than those of the $\mathrm{CO}_{2}$-biocathode, for which acetate may partly contribute electrons directly. This was consistent with the obviously higher CE of the ace-biocathode $(118.0 \pm 19.7 \%)$ than that of the $\mathrm{CO}_{2}$-biocathode $(78.6 \pm 4.6 \% ; P=0.004)$.

For the abiotic cathode, the $\mathrm{E}_{\mathrm{rNB}}(P<0.04)$ and $\mathrm{E}_{\mathrm{AN}}(P<0.01)$ within $24 \mathrm{~h}$ were both significantly lower than the ace-biocathode and $\mathrm{CO}_{2}$-biocahode modes. Even with a doubled batch time ( $\left.48 \mathrm{~h}\right)$ of the biocathode, NB still appeared in the abiotic catholyte $(0.023 \pm 0.010 \mathrm{mM})$, and the $\mathrm{E}_{\mathrm{AN}}$ was only $80.8 \pm 2.1 \%$. The poor $\mathrm{E}_{\mathrm{rNB}}$ and $\mathrm{E}_{\mathrm{AN}}$ of the abiotic cathode were accorded with previous results $[16,17]$. Meanwhile, the NOB occurred since the $6^{\text {th }}$ $\mathrm{h}$, with a maximum concentration of $0.035 \mathrm{mM}$ detected at the $24^{\text {th }} \mathrm{h}$, and was accumulated until the $36^{\text {th }} \mathrm{h}$. This was markedly in contrast with the biocathode modes, whose NOB was detected only in the first $3 \mathrm{~h}$ (data not shown). The current trends (Fig. 2B) also could explain the products difference between the abiotic cathode and biocathode modes. The maximum current of the abiotic cathode was much smaller than those of the ace-biocathode and $\mathrm{CO}_{2}$-biocahode modes. This strongly indicated the biofilm enhanced electron capture from the cathode and delivery for NB reduction. Besides, the ace-biocathode showed significantly or relatively higher reduction efficiency of NB to AN than those of the open circuit control (Fig. 2A and Table 1 ). The reduction rate constants of NB within $24 \mathrm{~h}$ were $0.65 \pm 0.17$ and $0.58 \pm 0.07 \mathrm{~h}^{-1}$ for the closed and open circuit ace-biocathode modes, respectively, which had no obviously difference. However, the constants for the first $6 \mathrm{~h}$ were $0.50 \pm 0.09$ and $0.22 \pm 0.05 \mathrm{~h}^{-1}$ respectively, which was significantly different $(P=0.05)$. After all, the transformation mainly occurred in the first $6 \mathrm{~h}$. The AN formation rates for the whole cycle were $0.49 \pm 0.05$ and $0.26 \pm 0.12 \mathrm{~h}^{-1}$ (nearly double; $P=0.03$ ) for the closed circuit ace-biocathode and open circuit mode, respectively, which together strongly indicated that the electrical stimulation obviously enhanced the reduction of NB to AN. This was in concert with the slow process of the pure anaerobic bioreduction. However, for the abiotic cathode without biofilm catalyst, NB was reduced merely by electrochemical reaction and did not show strong reduction ability. This further illustrated the indispensable catalytic function of $\mathrm{EAB}$, which markedly improved the reduction efficiency of $\mathrm{NB}$ to $\mathrm{AN}$ and avoided the accumulation of more toxic intermediate NOB.

\subsection{AO7 decolorization with the established biocathode}

After NB reduction, AO7 $(0.143 \pm 0.005 \mathrm{mM})$ as a model was selected to test the azo bond reduction with the established biocathode. The experimental parameters and designs kept similar with those in the NB reduction experiments. Each batch experiment lasted for $24 \mathrm{~h}$. Under the open circuit operation, AO7 was reduced mainly by anaerobic microbes with sodium acetate as electron donor. The $\mathrm{E}_{\mathrm{rAO}}$ could reach over $97.7 \pm 0.4 \%$ with the $E_{S A}$ of $73.5 \pm 4.1 \%$ within $24 \mathrm{~h}$. This result was comparable with the reported anaerobic bioreduction efficiency $[28,29]$. Once closed the circuit, the $E_{S A}$ was significantly elevated within the initial $12 \mathrm{~h}(P<0.02)$ and close to the value of open circuit control just at $24 \mathrm{~h}(P=0.26)$. Importantly, the transformation of A07 mainly occurred in the first $6 \mathrm{~h}$. The reaction rates were $0.44 \pm 0.03$ and $0.15 \pm 0.07 \mathrm{~h}^{-1}$ for the closed and open circuit ace-biocathodes respectively based on the SA formation (total 
$24 \mathrm{~h})$, which strongly indicated that they had obviously difference $(P=0.03)$. When doubled the AO7 influent concentration, the $\mathrm{E}_{\mathrm{rAO}}$ still kept over $97.2 \pm 0.1 \%$, illustrating the high catalytic efficiency of the ace-biocathode. Even when sodium bicarbonate replaced sodium acetate, the $\mathrm{E}_{\mathrm{rAO} 7}$ and $\mathrm{E}_{\mathrm{SA}}$ within $24 \mathrm{~h}$ had not been obviously influenced. However, the ace-biocathode had markedly higher $\mathrm{E}_{\mathrm{SA}}$ than those of the $\mathrm{CO}_{2}$-biocathode $(P<0.01)$ within the initial $12 \mathrm{~h}$ (Fig. $2 \mathrm{C}$ and Table 1 ). This may result from the additional electrons from sodium acetate and relatively slow carbon source supply from inorganic sodium bicarbonate. The abiotic cathode had the lowest AO7 to SA transformation efficiency. The discrepancy indicated the efficient catalyst of microbes for AO7 reduction. This was in accordance with the counterpart current trends (Fig. 2D). A previous study had presented the importance of organic carbon sources for the acclimatized anaerobic microbes to guarantee the azo dye reduction function [30]. Nevertheless, biofilms from polarity inversion strategy seemed to be not, the outperformance of the two biocathode modes showed the vivid function. Besides, it is reported that one of the AO7 reduction products 1-amino-2naphthol could work as electron mediator [29]. Once accumulated, the electron transfer seemed to be facilitated if there had sufficient electrons. However, without the microbial catalyst, the SA yield was very low $(0.064 \pm 0.004 \mathrm{mM})$ (Fig. $2 \mathrm{C})$. The result clearly depicted that the biofilm contributed greatly to electron transfer and SA formation. Furthermore, the electrical stimulation enhanced the SA formation efficiency. However, the azo bond cleavage products could easily undergo auto-oxidation reactions to form colored polymeric compounds [31], resulting in the relatively low SA transformation efficiency in this study.

\subsection{Confirmation of the catalytic ability of the established biocathode}

In order to exclude the possibility of hydrogen evolution, another set of control experiments were conducted by positing the potential at $-0.40 \mathrm{~V}$. Just as reported, hydrogen can work as electron mediator between electrodes and microbes [32]. Thereafter, the $\mathrm{CO}_{2}$-biocathode mode at $-0.40 \mathrm{~V}$ was performed for NB reduction. A higher potential deteriorated the catalytic reduction of the abiotic cathode. On the one hand, the $\mathrm{E}_{\mathrm{rNB}}$ of the abiotic cathode at the tested time points within $24 \mathrm{~h}$ were markedly lower than those with the biofilm $(P<0.04)$; on the other hand, NB was continuously reduced to NOB and potential phenylhydroxylamine, making a significantly lower $\mathrm{E}_{\mathrm{AN}}$ for the abiotic cathode $(P<0.01)$. Even with a doubled batch time of the biocathode, the $E_{\mathrm{AN}}$ (at $48 \mathrm{~h}$ ) of the abiotic cathode was still obviously lower than that of the biocathode at $24 \mathrm{~h}(P=0.01)$. The NOB was produced until the $36^{\text {th }} \mathrm{h}$ in the abiotic cathode, in contrasting with $6 \mathrm{~h}$ in the biocathode (data not shown). This was in accordance with the current trends. The initial maximum current for the abiotic cathode was inferior to that with the biofilm (Fig. 3A). Supposing that hydrogen had participated in the electron transfer between the electrode and biofilm under a lower potential, the NB reduction would be prominently declined with the rising of potential. However, the elevation of potential from -0.45 to $-0.40 \mathrm{~V}$ had no obviously influence on the $\mathrm{E}_{\mathrm{rNB}-\max }$ and $\mathrm{E}_{\mathrm{AN}-\max }$ as well as the $\mathrm{CE}$ of the $\mathrm{CO}_{2}$-biocathode. The maximum current drop for the $-0.40 \mathrm{~V}$ mode was correspondingly lower than that of the $-0.45 \mathrm{~V}$ mode ( $0.26 \mathrm{vs}$. $0.42 \mathrm{~mA}$ ), which likely explained the difference in the AN yields at the initial stage. These results demonstrated the strong electron transfer ability of the inverted biofilm and the negligible involvement of potential hydrogen. The results also highlighted the possibility of the biocathodic treatment of aromatic pollutants-containing oligotrophic wastewaters based on the microbial electrode-respiration. Additionally, the comparison of $\mathrm{E}_{\mathrm{rNB}}$ and $\mathrm{E}_{\mathrm{AN}}$ between the live biocathode, sterilized biocathode and abiotic cathode under $-0.40 \mathrm{~V}$ further demonstrated the direct cat-

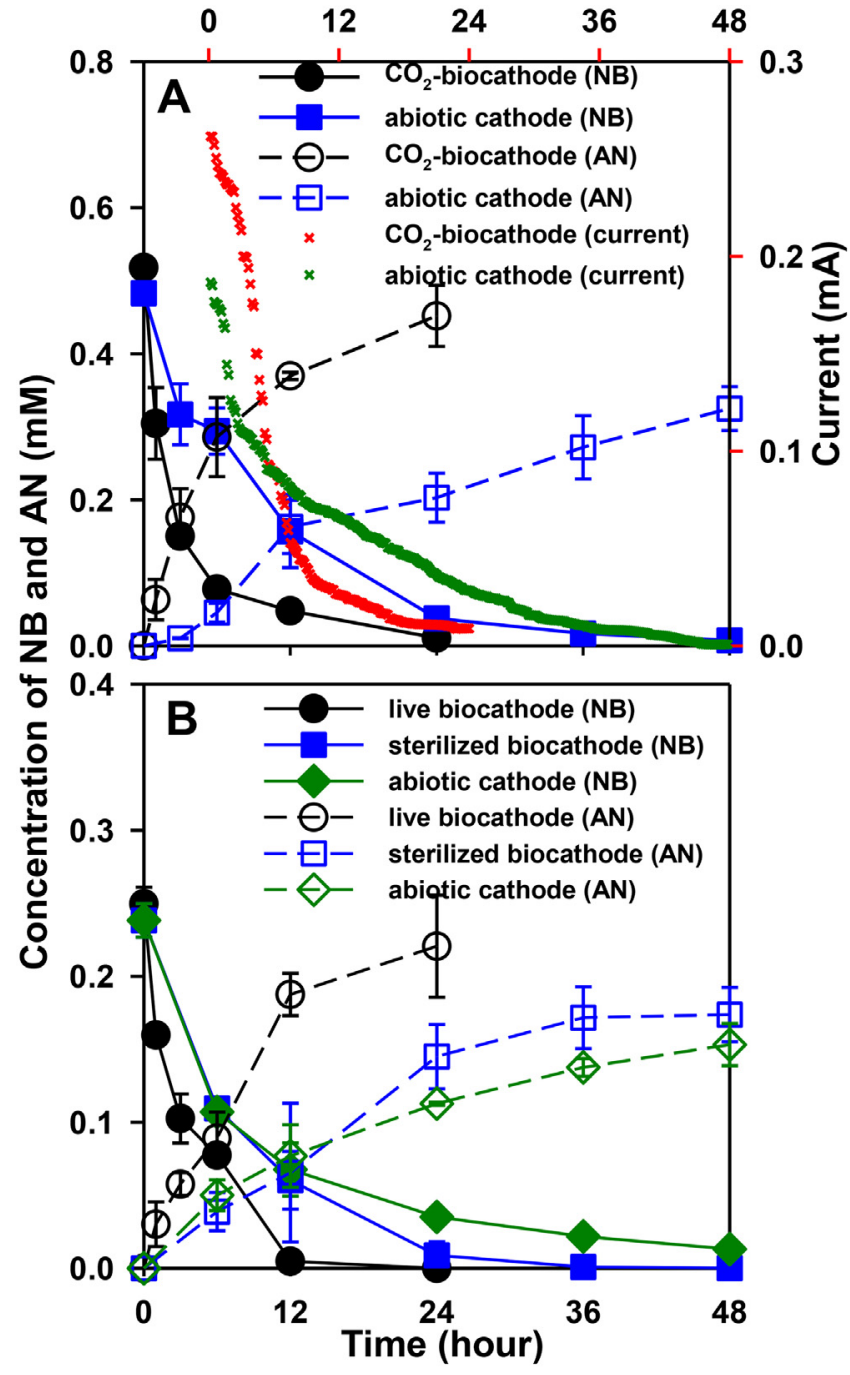

Fig. 3. The comparison of NB reduction under different conditions. (A): NB reduction and corresponding currents under $-0.40 \mathrm{~V}$ (carbon brush); (B): NB reduction with the live and sterilized biocathodes under $-0.40 \mathrm{~V}$ (carbon cloth).

alytic role of the electrophilic biofilm in the cathode, which played a key role in enhancing the reduction of NB to AN (Fig. 3B).

The catalytic activity of the established biocathode was also demonstrated through CV analysis. NB was selected as the substrate and scanned in all experimental modes. As shown in Fig. 4A, no obvious reductive currents were recorded for the PBS blank control (i.e. the abiotic cathode without NB). In contrast with the blank control, there were 3 peaks for NB on the abiotic carbon cloth electrode, including the NB reduction (at $-0.54 \mathrm{~V}$ ) and the redox reaction of phenylhydroxylamine and NOB $(0.154$ and $0.091 \mathrm{~V})$, which were the intermediates during NB reduction [16]. In case of the biocathode, the CV profile was obviously different, where the peak potential of NB reduction was positively shifted by about $0.15 \mathrm{~V}$ alongside with the folded increase of peak current. This clearly indicated the cathodic biofilm participated into the electrochemical catalysis of NB reduction. When the biocathode was sterilized, the peak current of NB reduction dropped back and even inferior to that of the abiotic cathode. This could be attributed to the fact that the dead cathode biofilm likely inactivated the conductive electrode or the dead biofilm attached on the electrode amplified the mass transfer resistance [33], which further implied this catalysis depended on the microbial activity. Additionally, the CV of the biocathode was also performed in the absence of NB. As shown in 
Fig. 4B, two pairs of redox peaks respectively centered on $-0.21 \mathrm{~V}$ and $-0.13 \mathrm{~V}$ were observed, which can be compared with those obtained in Geobacter or its dominant anodic biofilm [34,35] and was in agree with that the cathodic biofilm was originally formed in the anode mode. These redox peaks were previously reported to be raised by various types of outer-membrane cytochrome $c$ that mediated the electron transfer from bacteria to the anode [34]. Nevertheless, as indicated by the CV of the biocathode with and without NB in this study, the onset of catalytic current aroused by NB reduction also likely corresponded to these redox peaks upon inverting to the cathode mode. Especially for the redox peak with more negative mid-point potential (at $-0.21 \mathrm{~V}$ ), the cathodic peak almost had the same onset potential as that of NB reduction, while the anodic peak disappeared in the presence of NB. This result well consisted with the participation of the mediator in the cathodic process $[36,37]$ and suggested the involved redox compound is capable of performing backward electron transfer (i.e. from electrode to the electron acceptor). However, it is still not clear whether the putative redox compound is capable of directly taking NB as the electron acceptor or needs to cooperate with other enzymes, such as nitroreductase. A recent study found the redox complex established by acetateoxidizing anodic biofilm can be switched to implement the electron transfer for catalyzing the reduction of nitrate [38].
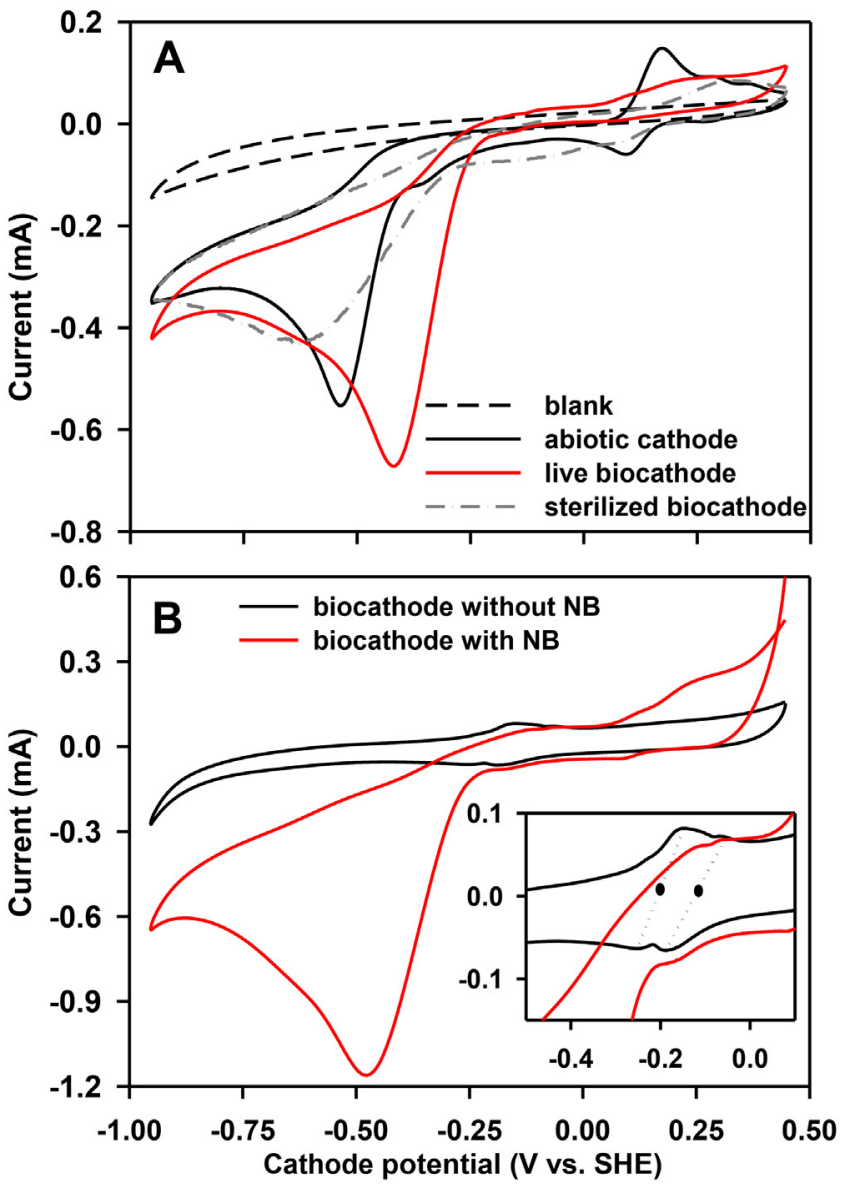

Fig. 4. Cyclic voltammograms of the abiotic cathode, live and sterilized biocathodes for NB reduction at scan rate of $2 \mathrm{mV} / \mathrm{s}(\mathrm{A})$. Cyclic voltammograms of the live biocathode with or without NB at scan rate of $10 \mathrm{mV} / \mathrm{s}$. Insert: enlarging for comparison of the live biocathode with or without NB in the potential window between 0.1 and $-0.5 \mathrm{~V}$ (B). Concentration of NB was $0.25 \mathrm{mM}$, if used.

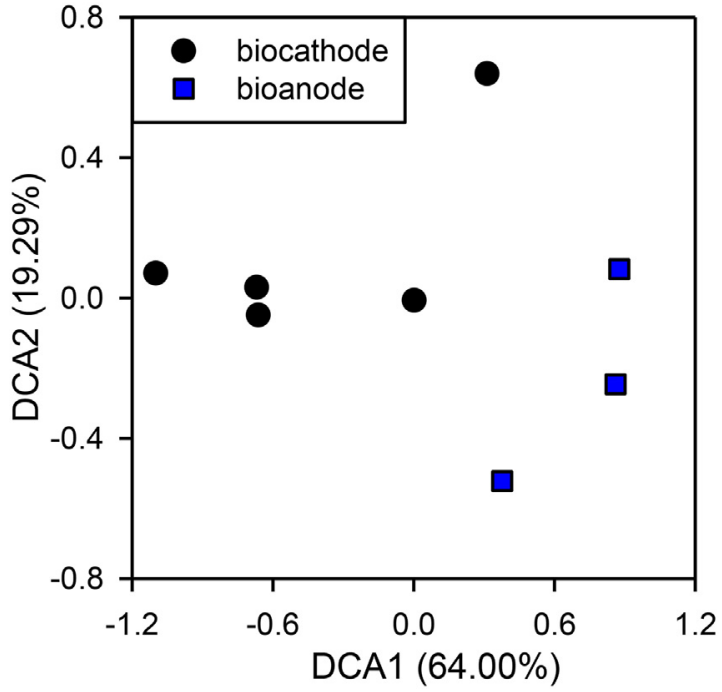

Fig. 5. Detrended correspondence analysis (DCA) of identified OTUs from $16 \mathrm{~S}$ rRNA gene sequencing showing that the direct polarity inversion significantly altered the bioanode communities structure and composition.

\subsection{Microbial communities structure and function}

In order to identify whether the polarity inversion would affect the phylogenetic structure and composition of these enriched bioanode communities, DCA was carried out with the relative abundance of identified OTUs (in total 1607). The DCA result showed that the established biocathode communities were well separated from the original bioanode communities by DCA1 axis (Fig. 5). The results of three nonparametric multivariate statistical approaches (ANOSIM, Adonis and MRPP) also strongly indicated that the biocathode communities structure significantly differed from the original bioanode communities ( 5 out of $6 P$ values $<0.05$, Table S1). The biodiversity indices richness, Chao 1 estimator, $H$, $1 / D$, Pielou and Simpson evenness were significantly $(P<0.05)$ or relatively decreased in the biocathode communities, indicating the polarity inversion obviously reduced the microbial phylogenetic diversity of the enriched biofilms (Table S2).

A total of 27 phyla were identified in these 8 electrode communities, therein 3 dominant phyla, including Proteobacteria, Bacteroidetes and Firmicutes, were frequently detected in electrode communities. Their abundances were not obviously different between the bioanode and biocathode groups, which illustrated the well maintenance of their electron transfer ability. After polar inversion, the relative abundance of Synergistetes (from $10.55 \pm 2.25$ to $29.82 \pm 13.78 \% ; P=0.03$ ) was markedly increased in the biocathode. While the bioanode enriched more Planctomycetes $(6.59 \pm 2.50 \% ; P=0.06)$ and Acidobacteria $(3.62 \pm 1.48 \% ; P=0.07)$ than the biocathode (Fig. S1). A total of 54 classes were classified and the most sequences belonged to 18 classes (relative abundance $>1 \%)$. Synergistia $(P=0.03)$, Methanobacteria $(P=0.09)$ and Clostridia $(P=0.04)$ species were increased in the biocathode compared to those in the bioanode, while Caldilineae, Planctomycetacia, Acidobacteria_Gp4 and other classified classes were obviously enriched in the bioanode (Fig. S1).

At the genus level, 309 genera were classified. Twenty-six genera with relative abundance $>0.5 \%$ were shown in Table 2 . For the biocathode communities established with traditional method (namely pre-enriched functional consortium working as inoculum of the biocathode), Geobacter populations were rarely enriched for nitroaromatics reduction $[8,16,17,39]$. Importantly, in this study, the abundance of the bidirectional electrode-respiring bacteria Geobacter had marginal difference before and after polar inversion 
Table 2

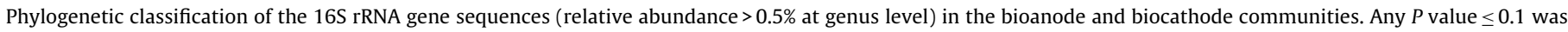
bolded.

\begin{tabular}{|c|c|c|c|c|c|c|}
\hline Phylum & Class & Family & Genus (\%) & bioanode & biocathode & $P$ value \\
\hline Synergistetes & Synergistia & Synergistaceae & Cloacibacillus & $9.73 \pm 2.27$ & $29.65 \pm 13.84$ & 0.03 \\
\hline Proteobacteria & $\delta$-proteobacteria & Geobacteraceae & Geobacter & $6.47 \pm 4.58$ & $6.06 \pm 1.97$ & 0.89 \\
\hline Proteobacteria & $\gamma$-proteobacteria & Moraxellaceae & Acinetobacter & $1.33 \pm 1.02$ & $1.88 \pm 0.41$ & 0.45 \\
\hline Proteobacteria & $\delta$-proteobacteria & Desulfovibrionaceae & Desulfovibrio & $1.41 \pm 0.47$ & $2.79 \pm 2.28$ & 0.25 \\
\hline Bacteroidetes & Bacteroidia & Porphyromonadaceae & Petrimonas & $0.38 \pm 0.13$ & $1.12 \pm 0.52$ & 0.03 \\
\hline Proteobacteria & $\gamma$-proteobacteria & Pseudomonadaceae & Pseudomonas & $0.17 \pm 0.06$ & $0.71 \pm 0.78$ & 0.20 \\
\hline Euryarchaeota & Methanomicrobia & Methanocorpusculaceae & Methanocorpusculum & $0.73 \pm 0.37$ & $1.58 \pm 0.76$ & 0.08 \\
\hline Euryarchaeota & Methanobacteria & Methanobacteriaceae & Methanobacterium & $0.33 \pm 0.23$ & $0.87 \pm 0.47$ & 0.07 \\
\hline Firmicutes & Clostridia & Clostridiales_Incertae Sedis XII & Acidaminobacter & $0.21 \pm 0.07$ & $0.67 \pm 0.69$ & 0.21 \\
\hline Acidobacteria & Acidobacteria_Gp4 & Unclassified & Gp4 & $2.78 \pm 1.37$ & $0.33 \pm 0.34$ & 0.09 \\
\hline Chloroflexi & Anaerolineae & Anaerolineaceae & Leptolinea & $0.88 \pm 0.45$ & $0.18 \pm 0.27$ & 0.10 \\
\hline Bacteroidetes & Bacteroidia & Porphyromonadaceae & Paludibacter & $1.20 \pm 0.44$ & $0.70 \pm 0.87$ & 0.32 \\
\hline Proteobacteria & Brproteobacteria & Rhodocyclaceae & Dechloromonas & $0.75 \pm 0.33$ & $0.45 \pm 0.56$ & 0.38 \\
\hline Chloroflexi & Caldilineae & Caldilineaceae & Caldilinea & $2.25 \pm 0.99$ & $0.38 \pm 0.25$ & 0.08 \\
\hline Chloroflexi & Anaerolineae & Anaerolineaceae & Longilinea & $0.95 \pm 0.40$ & $0.26 \pm 0.14$ & 0.08 \\
\hline TM7 & Unclassified & Unclassified & TM7 genera_incertae_sedis & $0.52 \pm 0.09$ & $0.29 \pm 0.13$ & 0.03 \\
\hline Planctomycetes & Planctomycetacia & Planctomycetaceae & Planctomyces & $1.62 \pm 0.49$ & $0.26 \pm 0.19$ & 0.03 \\
\hline Planctomycetes & Planctomycetacia & Planctomycetaceae & Zavarzinella & $1.15 \pm 0.49$ & $0.19 \pm 0.19$ & 0.08 \\
\hline Firmicutes & Bacilli & Pasteuriaceae & Pasteuria & $1.15 \pm 0.49$ & $0.24 \pm 0.09$ & 0.08 \\
\hline Armatimonadetes & Unclassified & Unclassified & Armatimonadetes_GP2 & $0.57 \pm 0.24$ & $0.31 \pm 0.13$ & 0.18 \\
\hline Planctomycetes & Planctomycetacia & Planctomycetaceae & Blastopirellula & $0.48 \pm 0.28$ & $0.12 \pm 0.09$ & 0.14 \\
\hline Proteobacteria & $\gamma$-proteobacteria & Methylococcaceae & Methylobacter & $0.67 \pm 0.36$ & $0.18 \pm 0.30$ & 0.13 \\
\hline Nitrospira & Nitrospira & Nitrospiraceae & Nitrospira & $0.55 \pm 0.27$ & $0.10 \pm 0.12$ & 0.09 \\
\hline Planctomycetes & Planctomycetacia & Planctomycetaceae & Rhodopirellula & $1.00 \pm 0.49$ & $0.13 \pm 0.16$ & 0.08 \\
\hline Euryarchaeota & Methanomicrobia & Methanosarcinaceae & Methanosarcina & $2.87 \pm 3.80$ & $1.32 \pm 1.33$ & 0.56 \\
\hline Euryarchaeota & Methanomicrobia & Methanosaetaceae & Methanosaeta & $2.81 \pm 1.24$ & $0.80 \pm 0.63$ & 0.09 \\
\hline
\end{tabular}

$(6.47 \pm 4.58 \%$ vs $6.06 \pm 1.97 \%)$. Geobacter not only has the ability of power production but also nitroaromatics/azo dye reduction [40]. Desulfovibrio, reputed to enable to grow with electron from electrode and to obtain electron for hydrogen production [41], had an increase abundance in the cathode $(2.79 \pm 2.28 \%)$. Another EAB, Pseudomonas also increased in the cathode $(0.71 \pm 0.78 \%)$. It is capable of secreting mediators, which could mediate and assist the electron transfer [42,43]. Collectively, Geobacter, Desulfovibrio and Pseudomonas which possess capabilities of bidirectional microbial electron transfer $[24,44,45]$ as well as nitroaromatics/azo dye bifunctional respiration [46-49] were maintained or enriched after the direct polarity inversion. Moreover, other bifunctional nitroaromatics/azo dye reducing microbes, including bacteria Acinetobacter $(1.88 \pm 0.41 \%)$ and archaea Methanobacterium $(0.87 \pm 0.47 \% ; P=0.07)$ [49-52] were enriched in the biocathode. The abundances of them in the anodic communities were $1.33 \pm 1.02 \%$ and $0.33 \pm 0.23 \%$ respectively. The aforementioned 5 genera species had abilities of nitroaromatics/azo dye bifunctional respiration and bioreduction in the biocathode communities, and this could be explained as the fact that bacterial azoreductase popularly worked as nitroreductase for the simultaneous reduction of nitroaromatics and azo dyes and vice versa [48,53-55]. This result suggests that the switchover of the electrons acceptor from NB to AO7 should have small but not significant effects on the diversity, structure and composition of biocathode communities. Cloacibacillus $(29.65 \pm 13.84 \% ; P=0.03)$, Petrimonas $(1.12 \pm 0.52 \% ; P=0.03)$ and Methanocorpusculum $(1.58 \pm 0.76 \%$; $P=0.08$ ) were enriched in the biocathode communities compared to those in the bioanode communities. Petrimonas was previously found in a NB-reducing biocathode with the traditional establishment method [16]. Cloacibacillus could potentially ferment amino acids from deciduous or dead biofilm cells to produce volatile acids such as acetate [56], which may be utilized by the EAB when the electrode worked as sole electrons donor for the biocathode communities. Additionally, 11 genera populations were significantly decreased after the polarity inversion (Table 2), which accorded with the obviously reduced biodiversity in the biocathode communities.

\subsection{Environmental implications}

Since biocathodes of BES have shown the great bioremediation potential, the rapid acclimation of non-specific functional biocathodes is critical for the treatment of complicated electron acceptors coexisting wastewaters. To our best knowledge, this is the first report about the direct polar inversion of traditional acclimated bioanode for the biocathodic reduction of aromatic pollutants. Unlike the traditional multistep enrichment process, the protocol of establishing a bioanode firstly and then switching to the cathode mentioned in this study can effectively shorten the establishment period (less than 2 weeks) without the loss of performances. The anode selection made the biofilm electrophilic and less specific, which could respond to different electron donors and acceptors and potentially widened the reducible substrates spectrum for the established biocathode. Organic and inorganic pollutants usually coexist in real wastewater or groundwater. Thus, it is promising for various organic and inorganic pollutants removal by the reductive metabolism of functional biocathodes. Previous studies indicated that the enriched nitrate or sulfate-reducing biocathode communities were also dominated by the EAB Geobacter species $[38,57,58]$. This further highlights the applicability of the proposed polarity inversion approach for the simultaneous treatment of organic and inorganic pollutants coexisting wastewater or groundwater. For one thing, high COD wastewaters like municipal wastewater, winery wastewater and mixed wastewater all have been used for the anodic biofilms acclimation and establishment [59-61]. The EAB Geobacter species was dominant as the anode biofilms enriched with sodium acetate in this study $[60,62,63]$. This strongly suggests that the practical municipal wastewaters could also serve as both inoculum and substrate to establish Geobacter species dominant anode biofilms. Such anode biofilms, possessing function of bidirectional electron transfer, provide prerequisite and feasibility for the reduction of nitro- and azo-aromatics in this work. For another, the developed biocathode in this way can enhance the reduction of the tested aromatic pollutants. Followed with the aerobic biodegradation, the daughter products from most aromatic compounds were considerably easier to be mineralized $[26,64,65]$. The mod- 
ular configuration may be introduced to establish and replenish electrochemically active biofilms with bidirectional electron transfer capability, for such cost-effective microbial catalyst was easy to be regenerated. Thus, the sustainable and simultaneous reduction of several environmental pollutants in a simple system could be realized. Furthermore, our results indicated that these biofilms from electrode selection had powerful electron transfer ability even using inorganic bicarbonate as carbon source. This is meaningful for some oligotrophic wastewaters or contaminated groundwater, where such biofilms are still capable of efficient reducing environmental pollutants independent of additional electrons donor, like organic carbon sources. Collectively, the current study offers new insights into the rapid establishment and modularization of functional biocathodes for the potential treatment of complicated electron acceptors coexisting wastewaters.

\section{Conclusions}

A novel method for the rapid establishment of non-specific functional biocathode communities by direct polar inversion of bioanode communities was developed. The biocathode could obviously accelerate the reduction of nitro- and azo-aromatics to their corresponding more biodegradable aromatic amines. Several electrode-respiring bacteria, possessing capabilities of nitro- and azo-aromatics reduction and bidirectional electron transfer were dominant in the biocathode communities. Geobacter containing cytochrome $c$ involved in the backward electron transfer from electrode to NB. This study provides an efficient strategy for the rapid establishment of non-specific functional biocathodes, which could be modularly established for the potential treatment of complicated electron acceptors coexisting wastewaters.

\section{Acknowledgments}

This work was supported by the National Natural Science Foundation of China (No. 31500084 and 21577162) and the Key Deployment Project of Chinese Academy of Sciences (No. ZDRWZS-2016-5-5).

\section{Appendix A. Supplementary data}

Supplementary data associated with this article can be found, in the online version, at http://dx.doi.org/10.1016/j.jhazmat.2017.02. 054.

\section{References}

[1] H. Wang, Z.J. Ren, A comprehensive review of microbial electrochemical systems as a platform technology, Biotechnol. Adv. 31 (2013) 1796-1807.

[2] M. Lu, S.F.Y. Li, Cathode reactions and applications in microbial fuel cells: a review, Crit. Rev. Environ. Sci. Technol. 42 (2012) 2504-2525.

[3] H. Wang, H. Luo, P.H. Fallgren, S. Jin, Z.J. Ren, Bioelectrochemical system platform for sustainable environmental remediation and energy generation, Biotechnol. Adv. 33 (2015) 317-334.

[4] J.S. Deutzmann, M. Sahin, A.M. Spormann, Extracellular enzymes facilitate electron uptake in biocorrosion and bioelectrosynthesis, mBio 6 (2015) e00496-00415.

[5] X.-W. Liu, W.-W. Li, H.-Q. Yu, Cathodic catalysts in bioelectrochemical systems for energy recovery from wastewater, Chem. Soc. Rev. 43 (2014) 7718-7745.

[6] D. Kong, B. Liang, H. Yun, H. Cheng, J. Ma, M. Cui, A. Wang, N. Ren, Cathodic degradation of antibiotics: characterization and pathway analysis, Water Res. 72 (2015) 281-292.

[7] D. Kong, B. Liang, H. Yun, J. Ma, Z. Li, A. Wang, N. Ren, Electrochemical degradation of nitrofurans furazolidone by cathode: characterization, pathway and antibacterial activity analysis, Chem. Eng. J. 262 (2015) 1244-1251

[8] B. Liang, H.-Y. Cheng, D.-Y. Kong, S.-H. Gao, F. Sun, D. Cui, F.-Y. Kong, A.-J. Zhou, W.-Z. Liu, N.-Q. Ren, Accelerated reduction of chlorinated nitroaromatic antibiotic chloramphenicol by biocathode, Environ. Sci. Technol. 47 (2013) 5353-5361.
[9] Y. Mu, K. Rabaey, R.A. Rozendal, Z. Yuan, J. Keller, Decolorization of azo dyes in bioelectrochemical systems, Environ. Sci. Technol. 43 (2009) 5137-5143.

[10] J. Radjenović, M.J. Farré, Y. Mu, W. Gernjak, J. Keller, Reductive electrochemical remediation of emerging and regulated disinfection byproducts, Water Res. 46 (2012) 1705-1714.

[11] M.H. Cui, D. Cui, L. Gao, A.J. Wang, H.Y. Cheng, Azo dye decolorization in an up-flow bioelectrochemical reactor with domestic wastewater as a cost-effective yet highly efficient electron donor source, Water Res. 105 (2016) 520-526.

[12] D. Cui, Y.Q. Guo, H.Y. Cheng, B. Liang, F.Y. Kong, H.S. Lee, A.J. Wang, Azo dye removal in a membrane-free up-flow biocatalyzed electrolysis reactor coupled with an aerobic bio-contact oxidation reactor, J. Hazard. Mater. 239-240 (2012) 257-264.

[13] A.J. Wang, D. Cui, H.Y. Cheng, Y.Q. Guo, F.Y. Kong, N.Q. Ren, W.M. Wu, A membrane-free continuously feeding, single chamber up-flow biocatalyzed electrolysis reactor for nitrobenzene reduction, J. Hazard. Mater. 199-200 (2012) 401-409.

[14] S.H. Gao, L. Peng, Y.W. Liu, X. Zhou, B.J. Ni, P.L. Bond, B. Liang, A.J. Wang, Bioelectrochemical reduction of an azo dye by a Shewanella oneidensis MR-1 formed biocathode, Int. Biodeter. Biodegr. 115 (2016) 250-256.

[15] X. Jiang, J. Shen, Y. Han, S. Lou, W. Han, X. Sun, J. Li, Y. Mu, L. Wang, Efficient nitro reduction and dechlorination of 2,4-dinitrochlorobenzene through the integration of bioelectrochemical system into upflow anaerobic sludge blanket: a comprehensive study, Water Res. 88 (2016) 257-265.

[16] B. Liang, H. Cheng, J.D. Van Nostrand, J. Ma, H. Yu, D. Kong, W. Liu, N. Ren, L. Wu, A. Wang, Microbial community structure and function of nitrobenzene reduction biocathode in response to carbon source switchover, Water Res. 54 (2014) 137-148.

[17] A.-J. Wang, H.-Y. Cheng, B. Liang, N.-Q. Ren, D. Cui, N. Lin, B.H. Kim, K. Rabaey, Efficient reduction of nitrobenzene to aniline with a biocatalyzed cathode, Environ. Sci. Technol, 45 (2011) 10186-10193.

[18] K.Y. Cheng, M.P. Ginige, A.H. Kaksonen, Ano-cathodophilic biofilm catalyzes both anodic carbon oxidation and cathodic denitrification, Environ. Sci. Technol. 46 (2012) 10372-10378.

[19] K.Y. Cheng, G. Ho, R. Cord-Ruwisch, Anodophilic biofilm catalyzes cathodic oxygen reduction, Environ. Sci. Technol. 44 (2010) 518-525.

[20] J.S. Geelhoed, A.J. Stams, Electricity-assisted biological hydrogen production from acetate by Geobacter sulfurreducens, Environ. Sci. Technol. 45 (2011) $815-820$.

[21] J.M. Pisciotta, Z. Zaybak, D.F. Call, J.Y. Nam, B.E. Logan, Enrichment of microbial electrolysis cell biocathodes from sediment microbial fuel cell bioanodes, Appl. Environ. Microbiol. 78 (2012) 5212-5219.

[22] K.Y. Cheng, G. Ho, R. Cord-Ruwisch, Novel methanogenic rotatable bioelectrochemical system operated with polarity inversion, Environ. Sci. Technol. 45 (2011) 796-802.

[23] H. Yun, D. Kong, B. Liang, M. Cui, Z. Li, A. Wang, Response of anodic bacterial community to the polarity inversion for chloramphenicol reduction, Bioresour. Technol. 221 (2016) 666-670.

[24] D.R. Lovley, Powering microbes with electricity: direct electron transfer from electrodes to microbes, Environ. Microbiol. Rep. 3 (2011) 27-35.

[25] D. Cui, F.-Y. Kong, B. Liang, H.-Y. Cheng, D. Liu, Q. Sun, A.-J. Wang, Decolorization of azo dyes in dual-chamber bioelectrochemical systems seeding with enriched inoculum, J. Environ. Anal. Toxicol. 001 (S3) (2011) (2012).

[26] D. Brown, P. Laboureur, The aerobic biodegradability of primary aromatic amines, Chemosphere 12 (1983) 405-414.

[27] H.Y. Cheng, B. Liang, Y. Mu, M.H. Cui, K. Li, W.M. Wu, A.J. Wang, Stimulation of oxygen to bioanode for energy recovery from recalcitrant organic matter aniline in microbial fuel cells (MFCs), Water Res. 81 (2015) 72-83.

[28] S.-A. Ong, E. Toorisaka, M. Hirata, T. Hano, Decolorization of Orange II using an anaerobic sequencing batch reactor with and without co-substrates, J. Environ. Sci. 24 (2012) 291-296.

[29] D. Méndez-Paz, F. Omil, J. Lema, Anaerobic treatment of azo dye Acid Orange 7 under fed-batch and continuous conditions, Water Res. 39 (2005) 771-778.

[30] S.-Y. Kim, J.-Y. An, B.-W. Kim, The effects of reductant and carbon source on the microbial decolorization of azo dyes in an anaerobic sludge process, Dyes Pigm. 76 (2008) 256-263.

[31] M. Kudlich, M.J. Hetheridge, H.-J. Knackmuss, A. Stolz, Autoxidation reactions of different aromatic o-aminohydroxynaphthalenes that are formed during the anaerobic reduction of sulfonated azo dyes, Environ. Sci. Technol. 33 (1999) 896-901.

[32] J. Li, G. Liu, R. Zhang, Y. Luo, C. Zhang, M. Li, Electricity generation by two types of microbial fuel cells using nitrobenzene as the anodic or cathodic reactants, Bioresour. Technol. 101 (2010) 4013-4020.

[33] L. Huang, Q. Wang, L. Jiang, P. Zhou, X. Quan, B.E. Logan, Adaptively evolving bacterial communities for complete and selective reduction of $\mathrm{Cr}$ (VI) $\mathrm{Cu}$ (II), and Cd (II) in biocathode bioelectrochemical systems, Environ. Sci. Technol. 49 (2015) 9914-9924.

[34] K.P. Katuri, P. Kavanagh, S. Rengaraj, D. Leech, Geobacter sulfurreducens biofilms developed under different growth conditions on glassy carbon electrodes: insights using cyclic voltammetry, Chem. Commun. 46 (2010) 4758-4760.

[35] S.A. Patil, F. Harnisch, C. Koch, T. Hübschmann, I. Fetzer, A.A. Carmona-Martínez, S. Müller, U. Schröder, Electroactive mixed culture derived biofilms in microbial bioelectrochemical systems: the role of $\mathrm{pH}$ on 
biofilm formation, performance and composition, Bioresour. Technol. 102 (2011) 9683-9690

[36] S. Freguia, S. Tsujimura, K. Kano, Electron transfer pathways in microbial oxygen biocathodes, Electrochim. Acta 55 (2010) 813-818.

[37] C. Coelho, J. Marangon, D. Rodrigues, J.J. Moura, M.J. Romão, P.M.P. de Sousa, M.M.C. dos Santos, Induced peroxidase activity of haem containing nitrate reductases revealed by protein film electrochemistry, J. Electroanal. Chem. 693 (2013) 105-113.

[38] N. Pous, A.A. Carmona-Martínez, A. Vilajeliu-Pons, E. Fiset, L. Bañeras, E. Trably, M.D. Balaguer, J. Colprim, N. Bernet, S. Puig, Bidirectional microbial electron transfer: switching an acetate oxidizing biofilm to nitrate reducing conditions, Biosens. Bioelectron. 75 (2016) 352-358.

[39] B. Liang, D. Kong, J. Ma, C. Wen, T. Yuan, D.-J. Lee, J. Zhou, A. Wang, Low temperature acclimation with electrical stimulation enhance the biocathode functioning stability for antibiotics detoxification, Water Res. 100 (2016) 157-168

[40] X. Jiang, J. Shen, S. Lou, Y. Mu, N. Wang, W. Han, X. Sun, J. Li, L. Wang, Comprehensive comparison of bacterial communities in a membrane-free bioelectrochemical system for removing different mononitrophenols from wastewater, Bioresour. Technol. 216 (2016) 645-652.

[41] S.M. Caffrey, H.S. Park, J. Been, P. Gordon, C.W. Sensen, G. Voordouw, Gene expression by the sulfate-reducing bacterium Desulfovibrio vulgaris Hildenborough grown on an iron electrode under cathodic protection conditions, Appl. Environ. Microbiol. 74 (2008) 2404-2413.

[42] E.M. Bosire, L.M. Blank, M.A. Rosenbaum, Strain- and substrate-dependen redox mediator and electricity production by Pseudomonas aeruginosa, Appl. Environ. Microbiol. 82 (2016) 5026-5038

[43] T. Zheng, Y.S. Xu, X.Y. Yong, B. Li, D. Yin, O.W. Cheng, H.R. Yuan, Y.C. Yong, Endogenously enhanced biosurfactant production promotes electricity generation from microbial fuel cells, Bioresour. Technol. 197 (2015) 416-421.

[44] B.E. Logan, Exoelectrogenic bacteria that power microbial fuel cells, Nat. Rev. Microbiol. 7 (2009) 375-381.

[45] W. Su, L. Zhang, D. Li, G. Zhan, J. Qian, Y. Tao, Dissimilatory nitrate reduction by Pseudomonas alcaliphila with an electrode as the sole electron donor, Biotechnol. Bioeng. 109 (2012) 2904-2910.

[46] E.S. Yoo, J. Libra, U. Wiesmann, Reduction of azo dyes by Desulfovibrio desulfuricans, Water Sci. Technol. 41 (2000) 15-22.

[47] M.J. Kwon, K.T. Finneran, Microbially mediated biodegradation of hexahydro-1,3,5-trinitro-1,3,5- triazine by extracellular electron shuttling compounds, Appl. Environ. Microbiol. 72 (2006) 5933-5941.

[48] G. Liu, J. Zhou, C. Chen, J. Wang, R. Jin, H. Lv, Decolorization of azo dyes by Geobacter metallireducens, Appl. Microbiol. Biotechnol. 97 (2013) 7935-7942.

[49] S. Mahmood, A. Khalid, M. Arshad, T. Mahmood, D.E. Crowley, Detoxification of azo dyes by bacterial oxidoreductase enzymes, Crit. Rev. Biotechnol. 36 (2016) 639-651.

[50] J.C. Spain, Biodegradation of nitroaromatic compounds, Annu. Rev. Microbiol. 49 (1995) 523-555.
[51] I. Soojhawon, P.D. Lokhande, K.M. Kodam, K.R. Gawai, Biotransformation of nitroaromatics and their effects on mixed function oxidase system, Enzyme Microb. Technol. 37 (2005) 527-533.

[52] F.J. Cervantes, A.B. Dos Santos, Reduction of azo dyes by anaerobic bacteria: microbiological and biochemical aspects, Rev. Environ. Sci. Biotechnol. 10 (2011) 125-137.

[53] F. Rafii, C.E. Cerniglia, Comparison of the azoreductase and nitroreductase from Clostridium perfringens, Appl. Environ. Microbiol. 59 (1993) 1731-1734.

[54] J. Rau, A. Stolz, Oxygen-insensitive nitroreductases NfsA and NfsB of Escherichia coli function under anaerobic conditions as lawsone-dependent Azo reductases, Appl. Environ. Microbiol. 69 (2003) 3448-3455.

[55] S.A. Misal, D.P. Lingojwar, M.N. Lokhande, P.D. Lokhande, K.R. Gawai, Enzymatic transformation of nitro-aromatic compounds by a flavin-free NADH azoreductase from Lysinibacillus sphaericus, Biotechnol. Lett. 36 (2014) 127-131.

[56] E. Jumas-Bilak, H. Marchandin, The phylum synergistetes, in: The Prokaryotes Springer, 2014, pp. 931-954

[57] W. Su, L. Zhang, Y. Tao, G. Zhan, D. Li, D. Li, Sulfate reduction with electrons directly derived from electrodes in bioelectrochemical systems, Electrochem. Commun. 22 (2012) 37-40.

[58] K.B. Gregory, D.R. Bond, D.R. Lovley, Graphite electrodes as electron donors for anaerobic respiration, Environ. Microbiol. 6 (2004) 596-604.

[59] P. Pandey, V.N. Shinde, R.L. Deopurkar, S.P. Kale, S.A. Patil, D. Pant, Recent advances in the use of different substrates in microbial fuel cells toward wastewater treatment and simultaneous energy recovery, Appl. Energy 168 (2016) 706-723.

[60] J. Yu, J. Seon, Y. Park, S. Cho, T. Lee, Electricity generation and microbial community in a submerged-exchangeable microbial fuel cell system for low-strength domestic wastewater treatment, Bioresour. Technol. 117 (2012) $172-179$.

[61] D. Pant, G. Van Bogaert, L. Diels, K. Vanbroekhoven, A review of the substrates used in microbial fuel cells (MFCs) for sustainable energy production, Bioresour. Technol. 101 (2010) 1533-1543.

[62] T.P. Sciarria, A. Tenca, A. D’Epifanio, B. Mecheri, G. Merlino, M. Barbato, S. Borin, S. Licoccia, V. Garavaglia, F. Adani, Using olive mill wastewater to improve performance in producing electricity from domestic wastewater by using single-chamber microbial fuel cell, Bioresour. Technol. 147 (2013) 246-253.

[63] R.D. Cusick, P.D. Kiely, B.E. Logan, A monetary comparison of energy recovered from microbial fuel cells and microbial electrolysis cells fed winery or domestic wastewaters, Int. J. Hydrogen Energy 35 (2010) 8855-8861.

[64] D. Cui, Y.-O. Guo, H.-S. Lee, H.-Y. Cheng, B. Liang, F.-Y. Kong, Y.-Z. Wang, L.-P. Huang, M.-Y. Xu, A.-J. Wang, Efficient azo dye removal in bioelectrochemical system and post-aerobic bioreactor: optimization and characterization, Chem. Eng. J. 243 (2014) 355-363.

[65] N.C. Tan, A. van Leeuwen, E.M. van Voorthuizen, P. Slenders, F.X Prenafeta-Boldu, H. Temmink, G. Lettinga, J.A. Field, Fate and biodegradability of sulfonated aromatic amines, Biodegradation 16 (2005) 527-537. 